Harvey Rosen, Department of Mathematics, University of Alabama,

Tuscaloosa, AL 35487, e-mail: hrosen@mathdept.as.ua.edu

\title{
DARBOUX QUASICONTINUOUS FUNCTIONS
}

\begin{abstract}
Let $C(f)$ denote the set of points at which a function $f: I \rightarrow I$ is continuous, where $I=[0,1]$. We show that if a Darboux quasicontinuous function $f$ has a graph whose closure is bilaterally dense in itself, then $f$ is extendable to a connectivity function $F: I^{2} \rightarrow I$ and the set $I \backslash C(f)$ of points of discontinuity of $f$ is $f$-negligible. We also show that although the family of Baire class 1 quasicontinuous functions can be characterized by preimages of sets, the family of Darboux quasicontinuous functions cannot. An example is found of an extendable function $f: I \rightarrow \mathbb{R}$ which is not of Cesaro type and not quasicontinuous.
\end{abstract}

\section{Extensions}

We begin with the following definitions of classes of functions which could be stated for $\mathbb{R}$ instead of $I$ or $\mathbb{R}^{2}$ instead of $I^{2}$.

D: A Darboux function $f: I \rightarrow I$ maps connected sets to connected sets, and so it has the intermediate value property.

Conn: A connectivity function $F: I^{2} \rightarrow I$ has the graph of its restriction $F \mid C$ connected for each connected subset $C \subset I^{2}$. According to [15], [10], and [16], this is equivalent to

PC: $F: I^{2} \rightarrow I$ is peripherally continuous if for each $x \in I^{2}$ and all open sets $U$ with $x \in U$ and $V$ with $F(x) \in V$, there exists an open set $W$ containing $x$ such that $W \subset U$ and $F($ bd $W) \subset V$.

Ext: A function $g: I \rightarrow I$ is said to be extendable if there exists a connectivity function $G: I^{2} \rightarrow I$ such that $G(x, 0)=g(x)$ for all $x \in I$. For such

Key Words: Darboux function, quasicontinuous function, extendable connectivity function, Baire 1 function, characterizable by preimages, Cesaro type function

Mathematical Reviews subject classification: Primary 26A15; Secondary 54C30

Received by the editors October 23, 1997 
an extendable function $g$, we then say that a set $A \subset I$ is $g$-negligible if whenever $f: I \rightarrow I$ is such that $f=g$ on $I \backslash A$ and the graph of $f \mid A$ is a subset of the closure, $\bar{g}$, of the graph of $g$, then $f$ is extendable, too.

AC: Every open neighborhood in $I^{2}$ of the graph of an almost continuous function $f: I \rightarrow I$ contains the graph of a continuous function $g: I \rightarrow I$.

QC: We say $f: I \rightarrow I$ is quasicontinuous if for each $x \in I$ and open sets $U$ containing $x$ and $V$ containing $f(x)$, there exists a nonempty open set $W \subset U$ such that $f(W) \subset V$. That is, $f \mid C(f)$ is dense in the graph of $f$.

DIVP: An $f: I \rightarrow I$ has the dense intermediate value property if $f(A) \in \mathcal{D}_{0}=$ $\{D \cap J: D$ is dense in $I$ and $J$ is a nonempty interval or singleton $\}$ for every $A \in \mathcal{D}_{0}$.

CT: A function $f: I \rightarrow I$ is of the Cesaro type if there exist nonempty open sets $U$ and $V$ in $I$ such that for each $y \in V, f^{-1}(y)$ is dense in $U$. Note that this implies the graph of $f$ is somewhere dense in $I^{2}$.

Let $\ell_{x}=\{x\} \times I$. A function $f: I \rightarrow I$ has a closure that is bilaterally dense in itself if for each $x \in(0,1), \operatorname{cl}(f \mid(0, x)) \cap \ell_{x}=\operatorname{cl}(f \mid(x, 1)) \cap \ell_{x}$. It follows from [11] that for a Darboux function $f: I \rightarrow I, \bar{f} \cap \ell_{x}$ is a connected set for each $x \in I$, and $C(f)$ is a dense $G_{\delta}$ subset of $I$ if $f$ also has a $G_{\delta}$ graph. Of course, a function $f$ equals its graph $\{(x, f(x)): x \in I\} . \Pi_{1}$ and $\Pi_{2}$ denote the $x$-projection and $y$-projection, respectively, of $I^{2}$ onto $I$. In [9], Gibson and Reclaw give an example of a Darboux quasicontinuous function $f: I \rightarrow I$ whose graph is not connected, and in [8], Gibson and Natkaniec give an example of an almost continuous quasicontinuous function $f: I \rightarrow I$ which is not extendable. Examination of many other examples in the literature revealed that whenever a Darboux quasicontinuous function $f$ was not extendable, then the closure of its graph failed to be bilaterally dense in itself. The first theorem shows that this is always the case. Example 1 in [9] is quasicontinuous with closure bilaterally dense in itself, but is not Darboux. Kellum and Garrett's function $f: I \rightarrow[-1,1]$ in Example 1 of [12] is in AC, a $G_{\delta}$ set, but not of Baire class 1 . Letting $K$ denote the Cantor ternary set in $I$ and $J=\left\{e_{1}, e_{2}, e_{3}, \ldots\right\}$ the set of endpoints of the complementary intervals of $K$, they define

$$
f(x)= \begin{cases}\sin \frac{1}{(m-x)(n-x)} & \text { if } x \text { belongs to the component }(m, n) \text { of } I \backslash K \\ 1 & \text { if } x \in K \backslash J \\ \frac{1}{r} & \text { if } x=e_{r} .\end{cases}
$$


In [6], Gibson asks if this function is extendable. Since $f$ is Darboux, quasicontinuous, and has a closure bilaterally dense in itself, then according to the following result, $f$ is extendable and $K$ is $f$-negligible.

Theorem 1. If $g: I \rightarrow I$ is a Darboux quasicontinuous function whose graph has a closure that is bilaterally dense in itself, then $g$ is extendable, and $I \backslash C(g)$ is g-negligible.

Proof. We identify $I$ with the subset $I \times\{0\}$ of $I^{2}$. By a "triangle" $t$, we mean $t=\operatorname{int}\left(s^{2}\right)$ (the set theoretic interior of $s^{2}$ in the space $I^{2}$ ), where $s^{2}$ is a closed 2-simplex in $I^{2}$ with a 1-dimensional face lying in $I \times\{0\}$. The "base" $b$ of $t$ is $b=t \cap(I \times\{0\})$. For each positive integer $n$ and $0 \leq i \leq 2^{n}-1$, define $H\left(\frac{i}{2^{n}}, \frac{i+1}{2^{n}}\right)=\left\{x \in I: \bar{g} \cap \ell_{x}\right.$ meets both $I \times\left\{\frac{i}{2^{n}}\right\}$ and $\left.I \times\left\{\frac{i+1}{2^{n}}\right\}\right\}$. Each $H\left(\frac{i}{2^{n}}, \frac{i+1}{2^{n}}\right)$ is closed and nowhere dense in I, and

$$
\bigcup\left\{H\left(\frac{i}{2^{n}}, \frac{i+1}{2^{n}}\right): 0 \leq i \leq 2^{n}-1\right\} \subset \bigcup\left\{H\left(\frac{j}{2^{n+1}}, \frac{j+1}{2^{n+1}}\right): 0 \leq j \leq 2^{n+1}-1\right\} .
$$

For $n=1,2,3, \ldots$ and $0 \leq i \leq 2^{n}-1$, we let $T_{n, i}$ denote a finite collection of disjoint triangles $t_{j}$ of diameter $<\frac{1}{n}$ in $I^{2}$ whose bases $b_{j}$ form a finite collection $B_{n, i}$ of disjoint open intervals of $I \times\{0\}$ with endpoints denoted endpts $\left(b_{j}\right) \subset C(g) \cup\{0,1\}$ such that

$$
\begin{aligned}
& I \times\{0\}=\bigcup\left\{\operatorname{cl}\left(b_{j}\right): b_{j} \in B_{n, i}\right\}, \\
& T_{n, k} \text { is a refinement of } T_{n, i} \text { for } k>i, \\
& T_{n+1,0} \text { is a refinement of } T_{n, 2^{n}-1} \\
& B_{n, k} \text { is a refinement of } B_{n, i} \text { for } k>i, \\
& B_{n+1,0} \text { is a refinement of } B_{n, 2^{n}-1}, \text { and }
\end{aligned}
$$

if 0 or 1 is an endpoint of $b_{j}$, then $\operatorname{cl}\left(t_{j}\right)$

is a neighborhood of $(0,0)$ or $(1,0)$, respectively , in $I^{2}$.

Picture the elements of each $T_{n, i}$ arranged like adjacent teeth of a handsaw and the sawteeth of the next collection, which is either $T_{n, i+1}$ or $T_{n+1,0}$, constructed inside the sawteeth of $T_{n, i}$. 
Since $B_{n, i}$ is an open cover of $H\left(\frac{i}{2^{n}}, \frac{i+1}{2^{n}}\right)$ with mesh $<\frac{1}{n}$,

if $x \in I \backslash C(g)$, then there exist $n$ and $i$ such that $x \in H\left(\frac{i}{2^{n}}, \frac{i+1}{2^{n}}\right)$ in which case $x \in b_{j}$ or $x=0$ or $x=1$ and $x$ is an endpoint of some

(7) $b_{j} \in B_{n, i}$, and we may assume $B_{n, i}$ is constructed so that $g\left(\operatorname{endpts}\left(b_{j}\right) \backslash\{0,1\}\right) \in\left[\frac{i}{2^{n}}, \frac{i+1}{2^{n}}\right]$ because $g$ has a closure that is bilaterally dense in itself and $g \mid C(g)$ is dense in $g$.

We can define an extension $G: I^{2} \rightarrow I$ of $g$ so that for each $n$ and $i$, the variation of $G$ on $\operatorname{bd}\left(t_{j}\right)$ (the set theoretic boundary in $I^{2}$ ) is $<\frac{1}{n}$ for each $t_{j} \in T_{n, i}$,

if $x \in\left(I \backslash C(g) \cap H\left(\frac{i}{2^{n}}, \frac{i+1}{2^{n}}\right)\right.$ and $x \in b_{j} \in B_{n, i}$, then $G\left(\operatorname{bd}\left(t_{j}\right)\right) \subset\left[\min g(\operatorname{endpts}(b j)), \max g\left(\operatorname{endpts}\left(b_{j}\right)\right)\right]$, but if $x=0$ or 1 and $x=\operatorname{endpt}\left(b_{j}\right)$, then $G\left(\operatorname{bd}\left(t_{j}\right)\right)=g\left(\operatorname{endpts}\left(b_{j}\right) \backslash\{0,1\}\right)$. If $x \in C(g)$ and $x=0$ or 1 and $x=\operatorname{endpt}\left(b_{j}\right)$ for some $b_{j} \in B_{n, i}$, then $G\left(\operatorname{bd}\left(t_{j}\right)\right) \subset\left[\min g\left(\operatorname{endpts}\left(b_{j}\right)\right), \max g\left(\operatorname{endpts}\left(b_{j}\right)\right)\right]$, and

$$
G \text { is continuous on } I^{2} \cup\left\{\operatorname{cl}\left(t_{j}\right): t_{j} \in T_{n, i}\right\} .
$$

Here is how to obtain condition (8). Suppose $E$ denotes the set of endpoints of all intervals belonging to $B_{n, i-1}$ along with the endpoints of just those members $b_{j}$ of $B_{n, i}$ constructed as described in (7) which each contain at least one point of $H\left(\frac{i}{2^{n}}, \frac{i+1}{2^{n}}\right)$ and which together cover $H\left(\frac{i}{2^{n}}, \frac{i+1}{2^{n}}\right)$. Suppose $c$ and $d$ are consecutive points of $E$ such that no point of $H\left(\frac{i}{2^{n}}, \frac{i+1}{2^{n}}\right)$ lies between $c$ and $d$. Because of (7) and (9), we may as well suppose that if $c=0$, then $c \in C(g)$. Even though $|g(d)-g(c)|$ might not be a small value, a finite number of triangles of diameter $<\frac{1}{n}$ belonging to $T_{n, i}$ can be constructed as follows forming sawteeth from $c$ to $d$ so that the total variation of $G$ on the slanted sides of each of the triangles will be less than $\frac{1}{n}$. First choose a partition $P=\left\{x_{0}=c, x_{1}, x_{2}, \ldots, x_{k}=d\right\}$ of $[c, d]$ in $C(g)$ with norm less than $\frac{1}{n}$. Next, since $g$ is Darboux, for $m=1,2, \ldots, k$, there exists a 
finite (possibly very irregular) partition $P_{m}$ of $\left[x_{m-1}, x_{m}\right]$ such that $g \mid P_{m}$ is monotone and $|g(y)-g(x)|<\frac{1}{n}$ for each pair of consecutive points $x$ and $y$ in $P_{m} . P \cup \bigcup_{m=1}^{k} P_{m}$ partitions $[c, d]$ into subintervals whose interiors are to belong to $B_{n, i}$ and are bases of a sawtooth collection of triangles of diameter $<\frac{1}{n}$ that are to belong to $T_{n, i}$. Then the extension $G$ of $g$ can be defined to be piecewise linear and of total variation $<\frac{1}{n}$ on the slanted sides of each triangle in this collection. We now show that $G: I^{2} \rightarrow I$ is in PC and hence in Conn. We only have to check peripheral continuity on $I \times\{0\}$ because according to (10), $G$ is continuous on $I^{2} \backslash(I \times\{0\})$. Let $\epsilon>0$.

Case 1: $x \in I \backslash C(g)$. Then by (7) and (9), $G$ is peripherally continuous at $(x, 0)$.

Case 2: $x \in C(g)$ and $x$ is an endpoint of an interval belonging to some $B_{m, i}$. If $x=0$ or 1 , then by $(9), G$ is peripherally continuous at $(x, 0)$. Therefore suppose $x \neq 0,1$. Then $x$ is an endpoint of adjacent intervals $b_{j}$ and $b_{k}$ in $B_{n, p}$ for each $B_{n, p} \in\left\{B_{m, i}, B_{m, i+1}, \cdots, B_{m+1,0}, B_{m+1,1}, \cdots\right\}$. There exists an $n \geq m$ such that $\frac{2}{n}<\epsilon, t_{j} \cup t_{k}$ has diameter $<\frac{2}{n}$, and the variation of $G$ on bd $\left(t_{j} \cup t_{k}\right)$ is $<\frac{2}{n}$. Since by (10), $G$ restricted to $I^{2} \backslash\left\{\operatorname{cl}\left(t_{j}\right): t_{j} \in T_{n, p}\right\}$ is continuous at $(x, 0)$, there exists an open semicircular disk $D$ in $I^{2}$ having $(x, 0)$ at the center of its diameter and not containing the other vertices of $t_{j}$ and $t_{k}$ such that the diameter of the open neighborhood $W=t_{j} \cup t_{k} \cup D$ of $(x, 0)$ in $I^{2}$ is $<\frac{2}{n}$ and $\operatorname{diam}(\{G(x, 0)\} \cup G(b d(W)))<\frac{2}{n}<\epsilon$. This shows $G$ is peripherally continuous at $(x, 0)$.

Case 3: $x \in C(g)$ and $x$ is not an endpoint of any $b_{j}$ in any $B_{n, i}$. For each $n$ and $i$, there exists $b_{j} \in B_{n, i}$ such that $(x, 0) \in b_{j}$. Let $\left\{a_{j}\right\}$ be a sequence whose $j$ th term $a_{j}$ is an endpoint of $b_{j}$ in $C(g)$. Then $a_{j} \rightarrow x$ and $G\left(a_{j}, 0\right) \rightarrow G(x, 0)$. Since the variation of $G$ on $\operatorname{bd}\left(t_{j}\right)$ is $<\frac{1}{n}, G$ is peripherally continuous at $(x, 0)$.

To show $I \backslash C(g)$ is $g$-negligible, suppose $f: I \rightarrow I$ with $f=g$ on $C(g)$ and $f \mid(I \backslash C(g)) \subset \bar{g}$. Since $g \mid C(g)$ is dense in $g$ and since $f=g$ on $C(g), \bar{f}=\bar{g}$. We show that

$$
F(x, t)= \begin{cases}G(x, t) & \text { on } I^{2} \backslash((I \backslash C(g)) \times\{0\}) \\ f(x) & \text { on }(I \backslash C(g)) \times\{0\}\end{cases}
$$

is a peripherally continuous extension of $f . F$ is peripherally continuous at each point of $I^{2} \backslash((I \backslash C(g)) \times\{0\})$ because $F=G$ on this set, which contains $\mathrm{bd}(W)$ in case 2 and contains $\mathrm{bd}\left(t_{j}\right)$ in case 3 . Let $x \in I \backslash C(g), \epsilon>0$ and $\delta>0$. There exist an $n$ and $i$ such that $\frac{1}{n}<\delta$ and

$$
\left[\frac{i}{2^{n}}, \frac{i+1}{2^{n}}\right] \subset(F(x, 0)-\epsilon, F(x, 0)+\epsilon) \cap \Pi_{2}\left(\bar{g} \cap \ell_{x}\right) .
$$


Then for some $j, x \in b_{j} \in B_{n, i}$ and $F\left(\operatorname{bd}\left(t_{j}\right)\right)=G\left(\operatorname{bd}\left(t_{j}\right)\right) \subset\left[\frac{i}{2^{n}}, \frac{i+1}{2^{n}}\right] \subset$ $(F(x, 0)-\epsilon, F(x, 0)+\epsilon)$. Therefore $F$ is peripherally continuous at each point of $(I \backslash C(g)) \times\{0\}$, too, and so $I \backslash C(g)$ is $g$-negligible.

A function $f: I \rightarrow \mathbb{R}$ belongs to $\mathrm{B}_{1}^{*}$, the class Baire* 1 , if each perfect set in $I$ contains a portion on which the restriction of $f$ is continuous. In the space $\mathrm{DB}_{1}$ of Darboux Baire class 1 functions $f: I \rightarrow \mathbb{R}$ with the metric $d(f, g)=\min \{1, \sup |f(x)-g(x)|\}$ of uniform convergence, let $\mathcal{G}$ be the subspace of quasicontinuous functions, and let $\mathcal{G}_{0}$ be the subspace of quasicontinuous functions having closures that are bilaterally dense in themselves. In [5], Darji, Evans, and O'Malley show that $\mathcal{G}$ is closed and nowhere dense in $\mathrm{DB}_{1}$ and that $\mathrm{DB}_{1}^{*}$ is of the first category in $\mathcal{G}$. $\mathcal{G}_{0}$ is closed in $\mathrm{DB}_{1}$ and a proof similar to theirs would show that the subspace of $\mathrm{DB}_{1}^{*}$ consisting of functions whose closures are bilaterally dense in themselves is of first category in $\mathcal{G}_{0}$.

\section{Preimages}

For $\mathcal{A}, \mathcal{B} \subset \mathcal{P}(\mathbb{R})$, the family of all subsets of $\mathbb{R}$, let $C_{\mathcal{A}, \mathcal{B}}=\left\{f \in \mathbb{R}^{\mathbb{R}}\right.$ : for every $A \in \mathcal{A}, f(A) \in \mathcal{B}\}$ and $C_{\mathcal{A}, \mathcal{B}}^{-1}=\left\{f \in \mathbb{R}^{\mathbb{R}}\right.$ : for every $B \in \mathcal{B}, f^{-1}(B) \in$ $\mathcal{A}$ \}. A family $\mathcal{F}$ of real functions is characterizable by images of sets if $\mathcal{F}=$ $C_{\mathcal{A}, \mathcal{B}}$ and by preimages of sets if $\mathcal{F}=C_{\mathcal{A}, \mathcal{B}}^{-1}$ for some $\mathcal{A}, \mathcal{B} \subset \mathcal{P}(\mathbb{R})$. The class $\mathrm{QC}$ of all quasicontinuous functions is characterizable by preimages [13] but not by images [3]. We examine the classes $\mathrm{QC} \cap \mathrm{B}_{1}, \mathrm{QC} \cap \mathrm{DIVP}$, and $\mathrm{QC} \cap \mathrm{D}$.

Theorem 2. $Q C \cap B_{1}$ is characterizable by preimages of sets.

Proof. Let

$$
\begin{array}{r}
\mathcal{A}=\left\{A \subset \mathbb{R}: A \text { is an } F_{\sigma} \text { set and for every interval }(a, b) \text { meeting } A,\right. \\
\left.(a, b) \cap A \text { contains a somewhere dense } G_{\delta} \text { subset of } \mathbb{R}\right\}
\end{array}
$$

and let $\mathcal{B}$ be the family of all open intervals $(c, d)$ in $\mathbb{R}$. If $f \in \mathrm{B}_{1}$, then $A=f^{-1}(c, d)$ is an $F_{\sigma}$ set and if $f \in \mathrm{QC}$, then each nonempty set $(a, b) \cap$ $f^{-1}(c, d)$ contains a somewhere dense $G_{\delta}$ subset (of continuities of $f$ ). Therefore $\mathrm{QC} \cap \mathrm{B}_{1} \subset C_{\mathcal{A}, \mathcal{B}}^{-1}$. Now suppose $f \in C_{\mathcal{A}, \mathcal{B}}^{-1}$. Then for every $(a, b)$ and $(c, d)$, if $(a, b) \cap f^{-1}(c, d)$ is nonempty, then it contains a somewhere dense $G_{\delta}$ subset $G$ of $\mathbb{R}$. Since $f^{-1}(c, d)$ is an $F_{\sigma}$ set for each $(c, d) \in \mathcal{B}, f \in \mathrm{B}_{1}$ and therefore $C(f)$ is a dense $G_{\delta}$ set. By the Baire Category Theorem, $C(f) \cap G$ is a somewhere dense $G_{\delta}$ subset of $\mathbb{R}$. It follows that $f \in \mathrm{QC}$. 
In [4], Ciesielski and Natkaniec show that the class DIVP cannot be characterized by preimages. Their exact same proof verifies the next result because the functions $f_{0}$ and $f_{1}$ they construct there in DIVP are also in QC.

Theorem 3. $Q C \cap D I V P$ cannot be characterized by preimages of sets.

Theorem 4. $Q C \cap D$ cannot be characterized by preimages.

Proof. Assume, otherwise, that there exist $\mathcal{A}, \mathcal{B} \subset \mathcal{P}(\mathbb{R})$ such that $\mathrm{QC} \cap \mathrm{D}=$ $C_{\mathcal{A}, \mathcal{B}}^{-1}$. We may suppose that $\mathcal{A}=\left\{f^{-1}(B): f \in \mathrm{QC} \cap \mathrm{D}\right.$ and $\left.B \in \mathcal{B}\right\}$ and $\mathcal{B} \not \subset\{\emptyset, \mathbb{R}\}$. Let $B \in \mathcal{B} \backslash\{\emptyset, \mathbb{R}\}$. Let $\left\{d_{n}: n=0,1,2, \ldots\right\}$ be a dense sequence in $B$ and $\left\{e_{n}: n=0,1,2, \ldots\right\}$ be a dense sequence in $\mathbb{R} \backslash B$. $C$ denotes the Cantor ternary set, and $J_{n}$ denotes the union of closures of all the components of $I \backslash C$ with length $\frac{1}{3^{n+1}}$. $C$ is the union of disjoint $c$-dense subsets $C_{1}$ and $C_{2}$. Let $C_{0}$ be the set of the endpoints of all the intervals $J_{n}$. Put

$$
f_{0}(x)= \begin{cases}e_{n} & \text { if } x \in J_{2 n} \\ e_{0} & \text { if } x \in\left(C_{1} \backslash C_{0}\right) \cup(\mathbb{R} \backslash(0,1)) \\ d_{n} & \text { if } x \in J_{2 n+1} \\ & \text { takes on every value of } \mathbb{R} c \text {-many times on every nonempty } \\ & \text { relative subinterval }(a, b) \cap\left(C_{2} \backslash C_{0}\right) .\end{cases}
$$

Let $C_{3}=\left\{x \in C_{2} \backslash C_{0}: f_{0}(x) \in B\right\}$. Then $f_{0} \in \mathrm{QC} \cap \mathrm{D}$ and $f_{0}^{-1}(B)=$ $C_{3} \cup \bigcup_{n=0}^{\infty} J_{2 n+1} \in \mathcal{A}$. Notice $C_{3}$ is $c$-dense in $C_{2}$. Now define

$f_{1}(x)= \begin{cases}e_{n} & \text { if } x \in J_{2 n+1} \\ d_{0} & \text { if } x \in\left(C_{2} \backslash\left(C_{3} \cup C_{0}\right)\right) \cup(\mathbb{R} \backslash(0,1)) \\ d_{n} & \text { if } x \in J_{2 n} \\ & \text { takes on every value of } \mathbb{R} \text { on every nonempty relative sub- } \\ & \text { interval }(a, b) \cap\left(\left(C_{1} \backslash C_{0}\right) \cup C_{3}\right) \text { with } f_{1}\left((a, b) \cap\left(C_{1} \backslash C_{0}\right)\right)=B \\ & \text { and } f_{1}\left((a, b) \cap C_{3}\right)=\mathbb{R} \backslash B .\end{cases}$

Then $f_{1} \in \mathrm{QC} \cap \mathrm{D}$ and $f_{1}^{-1}(B)=\left(\mathbb{R} \backslash\left(C_{3} \cup C_{0}\right)\right) \cup_{n=0}^{\infty} J_{2 n} \in \mathcal{A}, \mathbb{R}=f_{0}^{-1}(B) \cup$ $f_{1}^{-1}(B)$ and $f_{0}^{-1}(B) \cap f_{1}^{-1}(B)=\emptyset .\{\emptyset, \mathbb{R}\} \subset \mathcal{A}$ because the constant functions are in QC $\cap$ D. Define $h \in \mathbb{R}^{\mathbb{R}}$ by $h(x)=i$ if $x \in f_{i}^{-1}(B)$ and $i=0,1$. Therefore $h \in C_{\mathcal{A}, \mathcal{B}}^{-1} \backslash \mathrm{D}$, a contradiction.

\section{$3 \quad \operatorname{Ext} \backslash(\mathrm{CT} \cup \mathrm{QC})$}

Smital and Stanova showed that there exists an almost continuous function $f: I \rightarrow \mathbb{R}$ which is in neither CT nor QC [14]. In [7], Gibson asked if there 
exists an extendable function $f: I \rightarrow \mathbb{R}$ which is in neither CT nor QC. We see the answer is yes by applying the next theorem to either of the following examples.

Example 1. Let $f: I \rightarrow I$ be Croft's function, which has the properties that $f$ is Darboux, Baire class 1 , and $f=0$ a.e. but not identically 0. (See p.12 in [2].) Let $E \neq \emptyset$ be that set of measure zero. Then $f^{-1}(0)=I \backslash E$ and $I \backslash E$ is dense in $I$.

Example 2. More generally, suppose $\emptyset \neq E \subset I$ with $E$ an $F_{\sigma}$ set bilaterally $c$-dense in itself and $I \backslash E$ dense in $I$. For example, $E$ could be a certain union of countably many Cantor sets. By Theorem 2.4 on p. 13 in [2], there exists a Darboux Baire class 1 function $f: I \rightarrow I$ such that $f^{-1}(0)=I \backslash E$.

Theorem 5. If $f: I \rightarrow I$ is a Darboux Baire class 1 function and $E$ is a set obeying $\emptyset \neq E \subset I, f^{-1}(0)=I \backslash E$ and $I \backslash E$ is dense in $I$, then $f \in \operatorname{Ext} \backslash(C T \cup Q C)$.

Proof. According to Brown, Humke, and Laczkovich [1], a Baire class 1 function $f$ is Darboux if and only if $f$ is extendable. The graph of a Baire class 1 function $f$ is nowhere dense in $I \times I$ and hence $f \notin \mathrm{CT}$. Since $E \neq \emptyset$, there exists $a \in E$. Since $f^{-1}(0)=I \backslash E, f(a)>0$. Therefore $f$ is not quasicontinuous at $a$ because $f(a)>0, f(I \backslash E)=0$, and $I \backslash E$ is dense in I.

\section{References}

[1] J. B. Brown, P. Humke and M. Laczkovich, Measurable Darboux functions, Proc. Amer. Math. Soc., 102 (1988), 603-610.

[2] A. M. Bruckner,Differentiation of real functions, Lecture Notes in Math., vol. 659, Springer-Verlag, Berlin, 1978.

[3] K. Ciesielski, D. Dikranjan, S. Watson,Functions characterized by images of sets, Colloq. Math., to appear.

[4] K. Ciesielski and T. Natkaniec, Darboux like functions that are characterizable by images, preimages and associated sets, Real Analysis Exch., 23, this issue.

[5] U. B. Darji, M. J. Evans and R. J. O'Malley, Some interesting small subclasses of the Darboux Baire 1 functions, Real Analysis Exch. 19 (199394), 328-331. 
[6] R. G. Gibson, Darboux functions with a perfect road, Real Analysis Exch. 15 (1989-90), 582-591.

[7] R. G. Gibson, Concerning a characterization of continuity, Real Analysis Exch. 22 (1996-97), 437-442.

[8] R. G. Gibson and T. Natkaniec, Darboux like functions, Real Analysis Exch. 22 (1996-97), 492-533.

[9] R. G. Gibson and I. Reclaw, Concerning functions with a perfect road, Real Analysis Exch. 19 (1993-94), 564-570.

[10] M. R. Hagan, Equivalence of connectivity maps and peripherally continuous transformations, Proc. Amer. Math. Soc. 17 (1966), 175-177.

[11] F. B. Jones and E. S. Thomas, Jr., Connected $G_{\delta}$ graphs, Duke Math. J. 33 (1966), 341-345.

[12] K. R. Kellum and B. D. Garrett, Almost continuous real functions, Proc. Amer. Math. Soc. 33 (1972), 181-184.

[13] A. Neubrunnova, On certain generalizations of the notion of continuity, Mat. Casopis Sloven. Akad. Vied 23 (1973), 374-380.

[14] J. Smital and E. Stanova, On almost continuous functions, Acta Math. Univ. Comen. 37 (1980), 147-155.

[15] J. Stallings, Fixed point theorems for connectivity maps, Fund. Math. 47 (1959), 249-263.

[16] G. T. Whyburn, Connectivity of peripherally continuous functions, Proc. Nat. Acad. Sci. USA 55 (1966), 1040-1041. 